Running Head: Adult Bilinguals and Theory of Mind

Adult Bilinguals Outperform Monolinguals in Theory of Mind Ester Navarro \& Andrew R. A. Conway

Claremont Graduate University

Author Note

Ester Navarro, Department of Psychology, Claremont Graduate University.

Correspondence concerning this article should be addressed to Ester Navarro Garcia, Department of Psychology, Claremont Graduate University, 150 E 10th St, Claremont, CA. Contact: ester.navarrogarcia@,cgu.edu 


\begin{abstract}
Theory of mind (ToM) is an essential ability for social competence and communication, and it is necessary for understanding behaviors that differ from our own (Premack and Woodruff, 1978). Research on bilingual children has reported that 3 and 4year-old bilinguals outperform monolinguals in ToM tasks. While research on ToM has traditionally focused on children's performance, adult bilinguals' ToM abilities are largely unexplored. Research suggests that adult bilinguals also might outperform monolinguals (Rubio-Fernandez and Glucksberg, 2012), nevertheless, this difference has yet to be established using a more robust methodology. Here, we tested bilingual and monolingual adults on the director task (Dumontheil, Apperly, \& Blakemore, 2010). Results showed that bilingual adults outperformed monolinguals in response to perspective-dependent trials of the director task, but not in response to control trials. This suggests that bilingualism is associated with individuals' ability to take into account the perspective of another person. In addition, the number of cultures that participants were exposed to, regardless of bilingualism, was also associated with ToM performance. Overall, the findings suggest that linguistic and cultural experience have an impact on ToM.
\end{abstract}

Keywords: theory of mind, bilingualism, social cognition. 


\section{Adult Bilinguals Outperform Monolinguals in Theory of Mind}

Theory of mind (ToM) refers to the ability to attribute mental states, such as beliefs, intentions, and desires to others and use them to predict and interpret behavior (Premack \& Woodruff, 1978). According to a robust body of research, healthy children begin developing ToM between the ages of 3 and 4 (Wellman, Cross, \& Watson, 2001; Wimmer \& Perner, 1983) and even though ToM is developed by late childhood, there is evidence that the interaction between ToM and other cognitive processes continues to mature and vary throughout the lifespan (Dumontheil, Blakemore, \& Apperly, 2010).

On average, children fail to pass ToM tasks that require them to take another person's perspective into account before age 4 . However, research has shown that bilingual children outperform their monolinguals counterparts on ToM tasks, even when verbal ability and age are accounted for (Kovacs, 2009; Goetz, 2003; Greenberg, Bellana \& Bialystok, 2013). This suggests that bilinguals might develop ToM differently from monolinguals, reflected in earlier performance. However, it is unclear whether the same is true for adults. There is evidence that ToM is cognitively effortful when adults need to inhibit their own perspective, leading to egocentric errors (Apperly, Back, Samson, \& France, 2008; Apperly \& Butterfill, 2009; Keysar, Lin, \& Barr, 2003; Birch \& Bloom, 2007; Mitchell, Curry, \& Ziegler, 2009). In addition, research has shown that it is possible that adult bilinguals also have a ToM advantage over monolinguals (Rubio-Fernandez \& Glucksberg, 2012), although further research is needed to establish the validity of this effect. Understanding whether bilingualism affects adults' ToM performance can help further explain the mechanisms and processes involved in ToM.

The goal of the current study was to examine bilingual and monolingual adults' performance in a ToM task compared to a control task. We predicted a small but significant 
effect showing that bilingual adults outperform monolinguals on ToM, but not on a task that does not require taking account of another person's perspective.

\section{Bilingualism and Theory of Mind}

To effectively navigate complex social interactions, it is necessary to comprehend that unobservable mental states influence human behavior. Theory of mind (ToM) is an essential ability for social competence and communication (Sperber \& Wilson, 1995), and it is necessary for understanding behaviors that differ from our own (Perner, 1988; Wellman, 1990). In addition, the ability to understand that mental states differ has been linked to improved problem-solving skills and executive control performance (Greenberg, Bellana, \& Bialystok, 2013; German \& Hehman, 2006), and failure to pass ToM tasks is considered a symptom of a number of social-cognitive disorders (Baron-Cohen, Leslie, \& Frith, 1986; Hughes \& Russell, 1993).

A large body of literature has focused on children's early development of ToM. In fact, much is known about children's ToM development. For instance, on average, typically developing children under the age of 3 do not pass ToM tasks compared to their 4-year-old counterparts, suggesting that 3-year-olds do not yet have the ability to reason about beliefs in a flexible and efficient manner (Avis \& Harris, 1991; Goetz, 2003; Naito, Komatsu, \& Fuke, 2004). After age 4, children tend to accurately pass ToM tasks; however, evidence shows that ToM ability develops gradually throughout childhood (e.g., Carpendale \& Chandler, 1996), continues to develop throughout adolescence, and is still subject to changes in adulthood (Dumontheil, Apperly, \& Blakemore, 2010). In addition, ToM development is closely tied to the development of other abilities, such as vocabulary and executive control (Astington \& Baird, 2005; German \& Hehman, 2005), indicating that ToM likely requires cognitive abilities to overcome conflicting situations and therefore that adults can also make ToM mistakes (Apperly \& Butterfill, 2009; Keysar, Link, \& Barr, 2003). In fact, even though ToM 
is often considered a full-fledged ability in adults, evidence from a number of studies has shown that adults do not always use this ability to guide their behavior (Keysar, Barr, Balin, \& Brauner, 2000; Keysar, Lin, \& Barr, 2003), indicating that adults sometimes fail to remember what other people know and instead rely on egocentric knowledge.

While adults do not always use their ToM effectively, a number of studies have focused on how different experiences affect ToM performance. Specifically, a number of studies have reported that 3 and 4 -year-old bilingual children outperform their monolingual counterparts in ToM (Kovacs, 2009; Goetz, 2003; Greenberg, Bellana \& Bialystok, 2013, Schroeder, 2018). These findings suggest that bilinguals might engage different processes than monolinguals when using ToM. However, the underlying mechanisms that support this effect are unclear. Some researchers suggest that bilinguals might have enhanced executive control due to the constant switching between, and inhibition of, both of their languages, which in turn translates in domain-general cognitive enhancement (e.g., Bialystok, 2009; Bialystok \& Craik, 2010). Proponents of this hypothesis suggest that cognitive enhancement allows bilingual children to outperform their monolingual peers in tasks of ToM (Carlson \& Moses, 2001; Bialystok \& Senman, 2004). This view is in line with the performance view of ToM (Leslie, 1994; Leslie, German, \& Polizzi, 2005; Scholl \& Leslie, 2001) that suggests that the resolution of complex ToM tasks requires the development of other cognitive processes, such as executive control.

A different line of research proposes that the differences in ToM performance among bilingual and monolingual children might instead arise from early development of metalinguistic awareness (i.e., the ability to reflect upon and manipulate language) (Friesen \& Bialystok, 2012; Gombert, 1990). Research shows that bilingual children tend to have a metalinguistic awareness advantage over monolingual children (see Friesen \& Bialystok, 2012), suggesting that bilingual children might use metalinguistic awareness when engaging 
ToM. This is consistent with the competence view of ToM (Wellman, Cross, \& Watson, 2001; Wellman \& Liu, 2004). According to this view, children undergo a mental conceptual change (i.e., update and revision of preconceived theories) as a result of encountering instances in which they cannot achieve their goals because of their lack of awareness of other people's mental states. Some researchers have suggested that encountering people who do not speak one of the languages that a bilingual child can speak forces bilingual children to reflect on their knowledge of what other people know (and do not know) earlier than monolinguals, who do not encounter this problem (Kovacs, 2009).

While this finding seems to be robust among children (for a meta-analysis, see Schroeder, 2018), there is little evidence that this effect remains in adults. To examine whether adult bilinguals outperform monolinguals in ToM, Rubio-Fernandez and Glucksberg (2012) tested college students' eye fixations on a false-belief task (Wimmer \& Perner, 1983). The researchers found that bilinguals had fewer fixations in the egocentric item than monolinguals, thus outperforming monolinguals in the false belief task. In addition, bilinguals outperformed monolinguals on the Simon task of inhibitory control. Finally, performance in the ToM task was correlated with performance in the Simon task for both groups. This led the researchers to concluded that one possible factor underlying bilinguals' ToM performance could be executive control. However, this study presented several methodological limitations that hinder the validity of the results. Specifically, participants only responded to one trial of the false-belief task, decreasing the reliability of the measure. In addition, participants only completed 40 trials of the Simon task, which is far fewer than the appropriate number of trials used to measure inhibitory control in adults (von Bastian, Souza, \& Gade, 2016). Finally, the conclusion that executive control was the reason why bilinguals outperformed monolinguals in the false belief task was based on the correlation between performance in the Simon task and the false belief task. However, given that both 
executive control and language ability are predictors of ToM (Astington \& Baird, 2005; German \& Hehman, 2005), the correlation between the two tasks does not necessarily indicate that the reason bilinguals outperform monolinguals is solely because they have increased cognitive control. For these reasons, it is unclear whether this effect is consistent and whether it could be replicable with paradigms more commonly used to examine adult ToM.

Specifically, Dumontheil, Apperly, and Blakemore (2010) developed the director task. The director task assesses (1) individuals' ability to take the perspective of another person (i.e., Level 1 perspective taking; Flavell, Everett, Croft \& Flavell, 1981), which is one of the main components of ToM, and (2) general executive control (i.e., maintaining a rule in mind while inhibiting prepotent responses). Dumontheil et al. found that performance in the director task improves steadily from childhood to adulthood. Importantly, they also found that adults' responses to the director task where more accurate than young adults' responses, while executive control showed no differences between the groups. This suggests that perhaps ToM and executive control develop in combination, but ToM continues to vary based other factors, such as being bilingual or being exposed to different cultures (Wu \& Keysar, 2007).

One specific factor that could underly bilinguals' ToM performance is metalinguistic awareness. For example, Diaz and Farrar (2018) conducted a longitudinal study where they tested monolingual and bilingual children on ToM, metalinguistic awareness, and executive control and tested them again after a year. The researchers found that metalinguistic awareness at time 1 predicted ToM performance in bilinguals at time 2, whereas executive control at time 1 predicted ToM performance in monolinguals at time 2. Diaz and Farrar's study suggests that, perhaps in addition to executive control, bilinguals might be using processes that differ from those used by monolinguals, allowing them to outperform them. 
This suggest that ToM might evolve differently based on an individual's experience and can, to varying extents, be the result of interconnected processes with both executive control and linguistic ability.

Given that ToM develops throughout the lifespan, it is relevant to explore the extent to which adult bilinguals present improved ToM performance compared to monolinguals, as well as the factors that could account for this effect.

\section{Method}

\section{Participants and Design}

The experiment was a 2 (Trial Type: experimental, control) x 2 (Condition: Director, No Director) x 2 (Language Group: Bilingual, Monolingual) mixed factorial design with trial type and condition as within-subjects variables and language group as between-subjects variable. An a priori power analysis was conducted to determine an appropriate sample size using $\mathrm{G}^{*}$ Power (Erdfelder, Faul, \& Buchner, 1996). We assumed an effect size of $\eta^{2}=.05$ for the three-way interaction, power $=.90$, and a correlation among repeated measures of 4 . The analysis suggested a minimum number of $n=31$ participants per group. The final number of participants included in the analysis was $N=78$. Participants were recruited from The Claremont Colleges. 37 self-reported bilinguals and 41 monolinguals were included the study.

For the purpose of this study, we were interested in identifying bilinguals who had regularly used at least two languages for most of their lives. To be classified as bilingual, participants had to meet these criteria: (1) speak at least one language (in addition to English) at a native level, (2) have spoken the L2 for most of their lives, and (3) use both languages regularly. Monolingual participants were included if they had little or no knowledge of a language other than English. All participants completed the LEAP-Q (Marian, Blumenfeld, \& Kaushanskaya, 2007) questionnaire of language experience to collect this information. 
Bilinguals' mean age of acquisition of the L2 was $M=7.97($ Median $=6)$ and the average use of the L2 was $56 \%$ of the time. Bilinguals had spent an average of 13.57 years in the L2 country. Bilinguals' mean age was $M=27.29(S D=9.15$, Median =26) and monolinguals' mean age was $M=25.48(S D=9.74$, Median $=21)$. A total of 54 participants were female $(28$ bilinguals, 26 monolinguals). The L2s in our sample were Spanish (13) Mandarin (7), Farsi (2), Japanese (1), Korean (2), Italian (1), Hindi (2), Bangla (1), Polish (1), Igbo (1), Ga (1), Indonesian (2), Armenian (2), and Estonian (1). 27\% of bilinguals reported speaking more than two languages; $62 \%$ were considered early bilinguals (i.e., they learned their L2 before age 10). Crucially, $73 \%$ of bilinguals reported that English was their dominant language (see Table 1) and $23 \%$ reported that they considered both of their languages equally dominant. All participants were immersed in the US at the time of the study. 24 monolinguals were exposed to a single culture, 11 were exposed to 2 cultures, and 6 were exposed to 3 or more cultures. 9 bilinguals were exposed to a single culture, 21 were exposed to 2 cultures, and 8 were exposed to 3 or more cultures ${ }^{2}$. Participants were compensated with $\$ 10$ for completing the study.

\section{Materials}

Director task. The Director task by Dumontheil, Apperly, and Blakemore (2010) was used in this study. The task was programmed in E-Prime 2.0 and was presented on a desktop computer. The task includes two conditions (Director, No Director) and two trial types

(Experimental, Control). The stimuli are set up in a $4 \times 4$ shelf containing eight different objects arranged in different positions (see Figure 1). In the Director Condition, an avatar called the Director is placed behind the shelf. Some of the compartments in the shelf are occluded from the Director's view so that only the participant can see those objects. The Director stood on the other side of the shelf and viewed the shelf from behind, so that only the objects in the open compartments were visible to the Director. The participant was asked 
to listen to the instructions that the Director gave her through a headset. On each trial, the Director asked the participant to move one of the objects in a specific direction (e.g., "move the pencil up", "move the car down"). The participant responded by clicking on the object and dragging it to the appropriate slot within the shelf. Accuracy and reaction times of the responses were recorded.

The Director condition requires participants to take into account the fact that the perspective of the director is different from them, and that they therefore cannot refer to certain objects. Fort this reason, the director task requires that participants use information derived from level-1 perspective taking to infer the perspective of the Director. This is a core component of ToM that allows individuals to make inferences about other people's knowledge and beliefs based on their visual access in order to predict and explain the person's behavior. Both the Director and No Director condition require various executive functions, such as inhibiting the prepotent response and manipulating information in mind (i.e., working memory). However, in the No Director task, participants did not have to keep the perspective of the other person in mind. Rather, participants had to follow a rule.

Conditions. In the Director condition, participants were asked to consider the perspective of the Director. To this end, participants were shown the shelf from the perspective of the director and were explicitly told that the Director could not see objects in the occluded compartments. This condition assesses theory of mind because the participant has to remember that the perspective of the Director is not the same as theirs. In the No Director condition, participants are shown the same shelf, but the Director is not behind it anymore. Instead, participants are given a strategy; participants are told to ignore all objects placed in the slots with grey backgrounds. This condition does not require theory of mind and instead requires the participant to inhibit prepotent information while keeping in mind a rule, therefore just requiring general executive control. 
Trial types. Experimental trials were trials where the participant had to take into account the perspective of the Director. Participants had to select the correct response (i.e., the target), which was an object in the grid that both the participant and the director could see, however in experimental trials the shelf also showed a competing object that could be the most appropriate response but only from the perspective of the participant (i.e., Panel (c) in Figure 1). To respond correctly, participants had to consider the Director's perspective and avoid clicking on the competing object that was only visible to them. In Control trials, the target object had a competitor but was always the best response from both perspectives and no competing object was included in one of the grey compartments (i.e., Panel (d) in Figure 1). Filler trials referred to objects in the shelf that had no competitor and were visible to both Director and participant. The No Director condition included the same three type of trials. Experimental and control trials were never shown in the same stimulus (i.e., shelf display) and they appeared in a random order throughout the task. Each stimulus was presented for 2 seconds before the first auditory instruction and there were three auditory instructions per stimulus. Participants responded to a total of eight control trials, eight experimental trials and 48 filler in each condition.

LEAP-Q. Participants were also administered the Language Experience and Proficiency Questionnaire (LEAP-Q) (Marian, Blumenfeld, \& Kaushanskaya, 2007). The questionnaire requires participants to report information regarding dominant language, language acquisition order, average time of use per each language, years living in a country where the language is spoken, and total number of languages spoken (see Table 1).

\section{Procedure}

All participants completed the LEAP-Q questionnaire first. Then, participants were given standardized instructions for the director task and were shown an example of the stimulus (Figure 1, panels (a)-(b)). In the Director condition, they were told that the director 
would give them a series of instructions about where to move different objects in the shelf. The participants were explicitly told that the Director did not have their own perspective and could not see the objects in the occluded slots. Participants were asked to consider the avatar as a real person. Participants were also shown a stimulus from the perspective of the Director and were given examples of objects both them and the Director could see and objects only the participant could see. The task did not begin until the participant confirmed they understood this. Before the task began, there were 3 practice trials where the participant had to move three objects that the Director indicated. Participants only received feedback for the practice trials. All participants were administered the Director condition first and the No Director condition second to avoid that participants use the strategy provided in the No Director condition to respond to trials in the Director Condition (Dumontheil, Apperly, \& Blakemore, 2010). The entire study took approximately 30 to 40 minutes.

\section{Results}

A 2 (trial type: experimental, control) x 2 (language group: bilingual, monolingual) $\mathrm{x}$ 2 (condition: director, no director) mixed factorial ANOVA was conducted on accuracy. As predicted, there was a significant three-way interaction, $F(1,76)=10.61, p=.002, \eta_{\mathrm{p}}{ }^{2}=.12$ (see Figure 2). In addition, there was a main effect of condition, $F(1,76)=61.57, p<.001$, $\eta_{\mathrm{p}}{ }^{2}=.45$, indicating that participants performed less accurately in the Director than in the No Director condition.

To better understand the three-way interaction, two follow-up ANOVAs were then conducted for each of the two conditions (Director and No Director). For each condition, a 2 (trial type: experimental, control) x 2 (language group: bilingual, monolingual) mixed factorial ANOVA was conducted.

In the Director condition, the interaction was significant $(F(1,76)=7.55, p=.007$, $\left.\eta_{\mathrm{p}}{ }^{2}=.09\right)$; that is, bilinguals outperformed monolinguals in experimental trials, $t(73.75)=$ - 
2.26, $p=.027, d=-.51$ (bilingual: $M=.55, S D=.34$; monolingual: $M=.39, S D=.31$ ), but not in control trials $t(67.72)=1.29, p>.10 d=.29$ (bilingual: $M=.89, S D=.12$; monolingual: $M=.92, S D=.10)$. In addition, there was a main effect of trial type $(F(1,76)=$ $\left.144.75, p<.001, \eta_{\mathrm{p}}^{2}=.66\right)$, indicating that overall participants responded more accurately to control trials $(M=.90, S D=.11)$ than to experimental trials $(M=.47, S D=.33), t(93.5)=$ $10.97, p<.001, d=1.76^{1}$.

In contrast, in the No Director condition, the interaction was not significant $(F(1,76)$ $=1.26, p>.10, \eta_{\mathrm{p}}{ }^{2}=.01$ ), indicating that there were no differences between bilinguals and monolinguals performance. There was a main effect of trial type, $(F(1,76)=36.21, p<$ $\left..001, \eta_{\mathrm{p}}{ }^{2}=.32\right)$, indicating that participants responded more accurately to control trials $(M=$ $.98, S D=.11)$ compared to experimental trials $(M=.74, S D=.36), t(80.3)=5.81, p<.001$.

In addition to the primary analyses, a post-hoc analysis was conducted to examine the possible effect of exposure to culture on ToM. Culture has been shown to have an effect on ToM (Wu \& Keysar, 2007), therefore if improved performance in ToM can be affected by different social and linguistic factors, it is possible that having more experience handling different cultures can also affect ToM, above and beyond bilingualism.

A 2 (trial type: experimental, control) x 2 (condition: director, no director) x 2 (culture exposure: one culture, two cultures) mixed factorial ANOVA was conducted on ToM accuracy. The number of participants with 3 or more cultures was too small to include in the analysis, therefore only participants who reported being exposed to $1(\mathrm{n}=31)$ or $2(\mathrm{n}=33)$ cultures were included. There was a significant interaction between culture and trial type, $F(1,62)=7.92, p=.006, \eta_{\mathrm{p}}^{2}=.11$, indicating that participants exposed to two cultures outperformed those exposed to only one culture in experimental compared to control trials. Importantly, this was observed in the Director condition $(t(60.27)=-3.19, p=.002, d=-.79$ 
(one culture: $M=.35$; two cultures: $M=.60)$, but not in the No Director condition $(t(61.30)=$ $-1.43, p=>.10, d=-.35$ (one culture: $M=.67$; two cultures: $M=.79$ ).

\section{Discussion}

The goal of this study was to explore performance differences in theory of mind (ToM) among bilingual and monolingual adults. The Director condition required participants to remember the perspective of the director, that is, the objects that he could and could not see, whereas the No Director condition does not require considering the perspective of another person. We found that all participants performed better in control compared to experimental trials and, specifically they performed better in the No Director compared to Director condition. Importantly, bilinguals outperformed monolinguals in accurate responses to experimental trials that required taking into account the other person's perspective (i.e., Director condition) but not for the control trials (i.e., No Director). The results support previous research showing that that bilingual adults exhibit better performance in ToM compared to monolinguals (Rubio-Fernandez \& Glucksberg, 2012). That is, bilinguals respond more accurately to ToM tasks where the perspective of another person must be taken into account.

This study expands previous research by providing behavioral evidence for improved ToM performance among bilingual adults compared to monolinguals using a task that involves taking into account another person's beliefs. While the current findings do not focus on the underlying mechanisms that influence ToM performance, based on previous research (Diaz \& Farrar, 2017), bilinguals might engage different processes to resolve ToM tasks compared to monolinguals, perhaps due to bilinguals' early experience with ToM. In fact, our results suggest that different socio-linguistic factors may interact with ToM performance. For example, our post-hoc analysis shows that exposure to more than one culture also affects ToM performance, regardless of bilingualism. These results are consistent with research 
showing that different experiences that involve perspective-taking, such as being bilingual, can affect ToM development (Diaz \& Farrar, 2017; Wu \& Keysar, 2007). As previous research has proposed, it is possible that improved metalinguistic awareness as a result of experience with language accounts for the effect of bilingualism on ToM (Diaz \& Farrar, 2017). Future research should further examine this possibility empirically.

In addition, the results of this study contribute to the body of literature that shows that adults make ToM errors when a person's perspective differs from their own (Dumontheil, Blakemore, \& Apperly, 2010) and are consistent with evidence from neuroimaging studies indicating that brain regions involved in state attribution develop until late adolescence (e.g., Giedd et al., 1999; Shaw et al., 2008), perhaps shaping how adults use ToM. All participants in the study understood the difference in perspective between them and the director, however a significant number of participants did not reflect this knowledge in their responses. This suggests that ToM is an effortful process and is prone to errors, despite being a fully developed ability.

This study is not without limitations. While bilinguals and monolinguals were thought to be equated in cognitive ability ${ }^{2}$, it is possible that differences existed that were not accounted for, hindering the results. In addition, because bilinguals self-reported their language proficiency, it is possible that they did not accurately indicate the extent to which they are proficient in each of their languages. Finally, due to the need to avoid strategy building, the order of the conditions in the director task could not be counterbalanced, perhaps influencing performance in later trials of the No Director task. However, given that there were only eight trials per condition, performance was not expected to be strongly influenced.

Overall, this research shows that it is likely that several factors influence ToM based on an individual's experience. While executive control has been shown to be a relevant factor 
for ToM performance (Carlson \& Moses, 2001; German \& Hehman, 2006) and is necessary to resolve tasks that involve perspective-taking (Rubio-Fernandez \& Glucksberg, 2012), it is possible that different life experiences, such as growing up bilingual, or growing up with different cultural influences, also affect the way individuals interpret the perspectives of others, above and beyond executive control. A priority of future work should be to further explore the underlying processes behind the differences in ToM performance between bilinguals and monolinguals, as well as other factors that could potentially influence ToM. By understanding the processes that contribute to ToM, we can attempt to clarify the competence-performance debate and add to current theories of ToM that do not consider bilingual populations (e.g., Apperly \& Butterfill, 2009; Schaafsma, Pfaff, Spunt, \& Adolphs, 2015). 


\section{References}

Apperly, I. A., \& Butterfill, S. A. (2009). Do humans have two systems to track beliefs and belief-like states?. Psychological review, 116(4), 953.

Apperly, I. A., Back, E., Samson, D., \& France, L. (2008). The cost of thinking about false beliefs: Evidence from adults' performance on a non-inferential theory of mind task. Cognition, 106(3), 1093-1108. https://doi.org/10.1016/j.cognition.2007.05.005

Astington, J., \& Baird, J. A. (Eds.). (2005). Why language matters for theory of mind. Oxford, United Kingdom: Oxford University Press.

Avis, J. \& Harris, P. L. (1991). Belief-desire reasoning among Baka children: Evidence for a universal conception of mind. Child Development, 62, 460-467.

Baron-Cohen, S., Leslie, A. M., \& Frith, U. (1985). Does the autistic child have a "theory of mind"? Cognition, 21, 37-46.

Baum, S., \& Titone, D. (2014). Moving toward a neuroplasticity view of bilingualism, executive control, and aging. Applied Psycholinguistics, 35(5), 857-894.

Bialystok, E. (1992). Selective attention in cognitive processing: The bilingual edge. In R. J. Harris (ed.), Cognitive processing in bilinguals, pp. 501-513. New York: Elsevier Science Publishers.

Bialystok, E. (1999). Cognitive complexity and attentional control in the bilingual mind. Child Development, 70, 636-644. doi: 10.1111/1467-8624.00046

Bialystok, E., \& Senman, L. (2004). Executive processes in appearance-reality tasks: The role of inhibition of attention and symbolic representation. Child development, 75, $562-579$.

Bialystok, E., Craik, F. I., \& Ryan, J. (2006). Executive control in a modified antisaccade task: Effects of aging and bilingualism. Journal of experimental psychology: Learning, Memory, and Cognition, 32, 1341-1354. 
Bialystok, E. (2009). Bilingualism: The good, the bad, and the indifferent. Bilingualism: Language and Cognition, 12, 3-11.

Birch, S. A. J., \& Bloom, P. (2007). The curse of knowledge in reasoning about false beliefs: Research report. Psychological Science, 18(5), 382-386. https://doi.org/10.1111/j.1467-9280.2007.01909.x

Carlson, S. \& Moses, L. (2001). Individual differences in inhibitory control and children's theory of mind. Child Development, 72, 1032-1053.

Diaz, V., \& Farrar, M. J. (2018). The missing explanation of the false-belief advantage in bilingual children: a longitudinal study. Developmental science, 1-13.

Dumontheil, I., Apperly, I. A., \& Blakemore, S. J. (2010). Online usage of theory of mind continues to develop in late adolescence. Developmental science, 13(2), 331-338.

Erdfelder, E., Faul, F., \& Buchner, A. (1996). GPOWER: A general power analysis program. Behavior research methods, instruments, \& computers, 28(1), 1-11.

Friesen, D. C., \& Bialystok, E. (2012). Metalinguistic Ability in Bilingual Children: The Role of Executive Control. Rivista di psicolinguistica applicata, 12(3), 47-56.

German, T. P., \& Hehman, J. A. (2006). Representational and executive selection resources in 'theory of mind': Evidence from compromised belief-desire reasoning in old age. Cognition, 101(1), 129-152.

Giedd, J.N., Blumenthal, J., Jeffries, N.O., Castellanos, F.X., Liu, H., Zijdenbos, A., Paus, T., Evans, A.C., \& Rapoport, J.L. (1999). Brain development during childhood and adolescence: a longitudinal MRI study. Nature Neuroscience, 2, 861-863.

Goetz, P. J. (2003). The effects of bilingualism on theory of mind development. Bilingualism: Language and Cognition, 6, 1-15. 
Greenberg, A., Bellana, B., \& Bialystok, E. (2013). Perspective-taking ability in bilingual children: Extending advantages in executive control to spatial reasoning. Cognitive development, 28, 41-50.

Hughes, C., \& Russell, J. (1993). Autistic children's difficulty with mental disengagement from an object: Its implications for theories of autism. Developmental Psychology. https://doi.org/10.1037//0012-1649.29.3.498

Keysar, B., Barr, D. J., Balin, J. A., \& Brauner, J. S. (2000). Taking perspective in conversation: The role of mutual knowledge in comprehension. Psychological Sciences, 11, 32-38.

Keysar, B., Lin, S., \& Barr, D. J. (2003). Limits on theory of mind use in adults. Cognition, 89, 25-41.

Kovács, Á. M. (2009). Early bilingualism enhances mechanisms of false-belief reasoning. Developmental science, 12, 48-54.

Lanza, E. (1992). Can bilingual two-year-olds code-switch? Journal of Child Language, 19, 633-658.

Marian, V., Blumenfeld, H. K., \& Kaushanskaya, M. (2007). The Language Experience and Proficiency Questionnaire (LEAP-Q): Assessing language profiles in bilinguals and multilinguals. Journal of Speech, Language, and Hearing Research.

Mitchell, P., Currie, G., \& Ziegler, F. (2009). Two routes to perspective: Simulation and ruleuse as approaches to mentalizing. British Journal of Developmental Psychology, 27(3), 513-543. https://doi.org/10.1348/026151008X334737

Naito, M., Komatsu, S., \& Fuke, T. (2004). Normal and autistic children's understanding of their own and others' false-belief: a study from Japan. British Journal of Developmental Psychology, 12, 403-416. 
Paap, K. (2019). The Bilingual Advantage Debate. In Schwieter, J.W. (Ed.), The Handbook of the Neuroscience of Multilingualism (701-735). New York: Willey.

Perner, J. (1988). Developing semantics for theories of mind: from propositional attitudes to mental representations. In J. Astington, P. Harris, \& D. Olson (Eds.), Developing theories of mind. New York: Cambridge University Press.

Premack, D., \& Woodruff, G. (1978). Does the chimpanzee have a theory of mind?. Behavioral and brain sciences, 1(4), 515-526.

Schaafsma, S. M., Pfaff, D. W., Spunt, R. P., \& Adolphs, R. (2015). Deconstructing and reconstructing theory of mind. Trends in Cognitive Sciences, 19(2), 65-72. https://doi.org/10.1016/j.tics.2014.11.007

Scholl, B.J., \& Leslie, A.M. (2001). Minds, modules, and meta- analysis. Child Development, 72, 696-701.

Schroeder, S. R. (2018). Do bilinguals have an advantage in theory of mind? A metaanalysis. Frontiers in Communication, 3, 36.

Shaw, P., Kabani, N.J., Lerch, J.P., Eckstrand, K., Lenroot, R., Gogtay, N., Greenstein, D., Clasen, L., Evans, A., Rapoport, J.L., Giedd, J.N., \& Wise, S.P. (2008). Neurodevelopmental trajectories of the human cerebral cortex. Journal of Neuroscience, 28, 3586-3594.

Sperber, D., \& Wilson, D. (1995). Relevance: Communication and cognition. Cambridge, MA: Harvard University Press.

Wellman, H. M. (1990). The child's theory of mind. Cambridge, MA: MIT Press.

Wellman, H.M., Cross, D., \& Watson, J. (2001). Meta-analysis of theory-of-mind development: the truth about false belief. Child Development, 72, 655-684. 
Wimmer, H. \& Perner, W. (1983). Beliefs about beliefs: Representation and constraining function of wrong beliefs in young children's understanding of deception. Cognition, 13, 103-128.

Wu, S., \& Keysar, B. (2007). The effect of culture on perspective taking. Psychological science, 18(7), 600-606. 


\section{${ }^{1}$ Footnote}

${ }^{1}$ Reaction times (RTs) were also analyzed. Responses to correct trials only were included in the analysis. Outliers were examined for bilinguals and monolinguals based on RTs above 3 SDs. Only complete cases were included in the analysis. After removing outliers, 31 bilinguals and 33 monolinguals were included in the analysis. As expected, there were no significant differences in reaction time for trial types or bilingual group for either condition as observed in previous research (Dumontheil, Apperly, \& Blakemore, 2010). However, there was a nonsignificant trend showing that bilinguals $(M=3089.7, \mathrm{SD}=$ 516.33) were slower than monolinguals $(M=2896.7, \mathrm{SD}=375.95)$ for experimental trials in the Director condition.

\section{${ }^{2}$ Footnote}

${ }^{2}$ Because all participants in the study were students from the Claremont Colleges, both bilingual and monolingual participants were recruited from a selective pool of students. For this reason, we did not expect the sample to present significant variation in IQ or verbal ability. 


\section{Acknowledgments}

We want to thank Ian Apperly for kindly sharing the Director Task materials. 


\section{Table 1}

Bilingual Participants Language Experience based on LEAP-Q ( $\mathrm{N}=37)$.

\begin{tabular}{l|l}
\hline Experiences & \\
\hline Age of acquisition of L2 & $M=7.97(S D=8.19)$ \\
\hline Speaks more than 2 languages & $27 \%$ \\
Learned L2 before age 10 & $62 \%$ \\
Learned L2 after age 10 & $38 \%$ \\
\hline Average daily L1 use & $40 \%$ \\
Average daily L2 use & $56 \%$ \\
Average daily L3 use & $4 \%$ \\
\hline English as dominant language & $73 \%$ \\
\hline Years spent living in L1 country & $19.27(S D=11.3)$ \\
\hline Years spent living in L2 country & $13.57(S D=10.3)$ \\
\hline Exposure to one culture & 24 monolinguals \\
& 9 bilinguals \\
\hline Exposure to two cultures & 11 monolinguals \\
\hline Exposure to three or more cultures & 21 bilinguals \\
\hline
\end{tabular}


(a) Instructions example 1

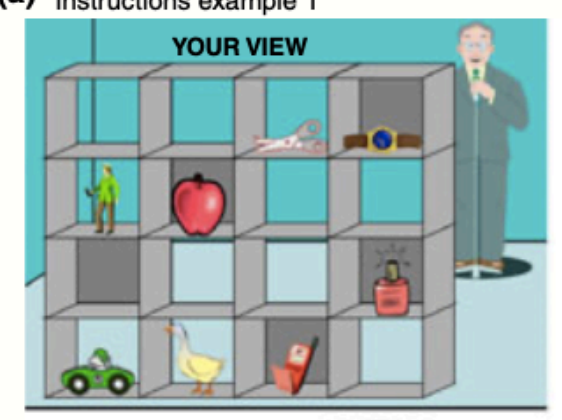

(c)

c) Experimental trial

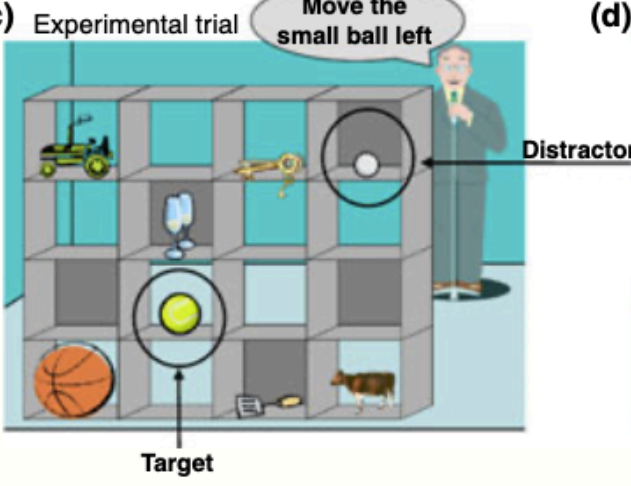

(b)

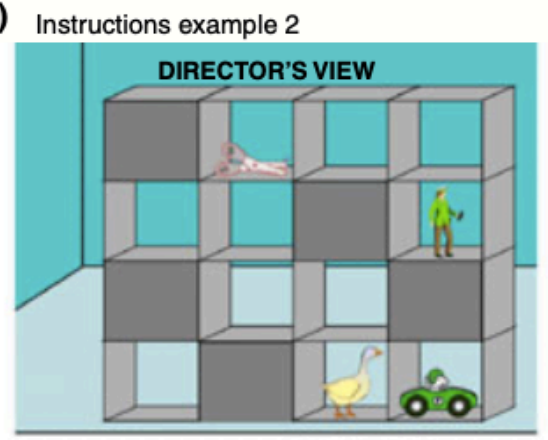

(d)

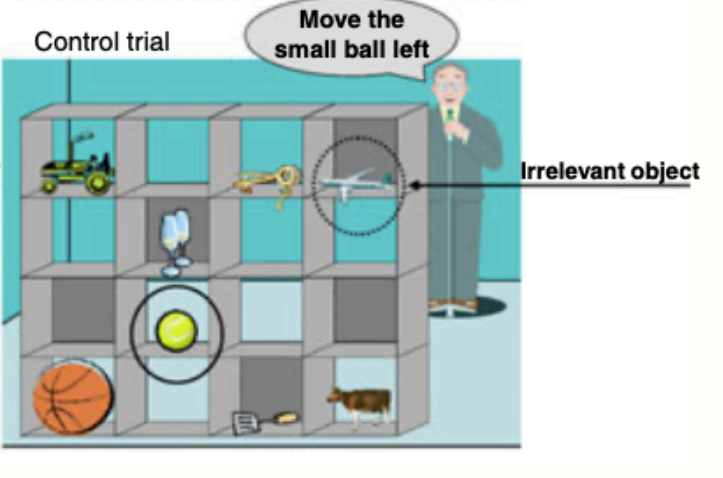

Figure 1. Director task (Dumontheil, Apperly, \& Blakemore, 2010). During the instructions phase, subjects were shown an example of their view (a) and the corresponding Director's view (b) for a given trial. During the experiment phase, subjects could encounter experimental trials (c) or control trials (d). Participants had to follow the oral instruction given by the Director. In experimental trials (c), the participant should move the target item (tennis ball) and ignore the distractor item (golf ball) if they took account of the Director's perspective. In control trials, an irrelevant object was shown instead of the distractor. Reprinted with Permission. 


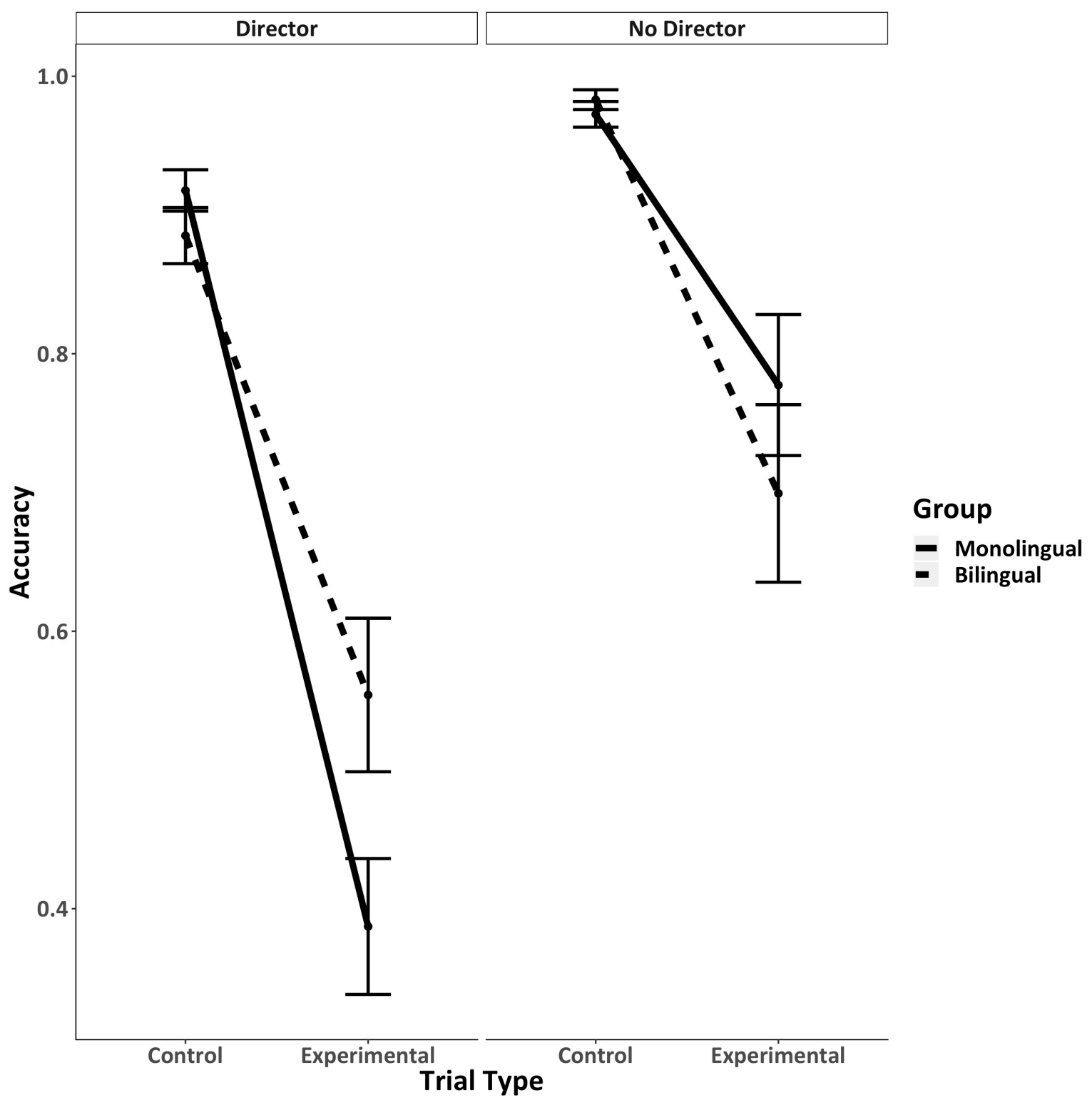

Figure 2. Accuracy by language group and trial type per condition. Bilingual participants outperformed monolinguals in accurate responses to experimental trials in the Director condition. 